\title{
Motivaciones y actitudes hacia la donación voluntaria de ovocitos. Experiencia de la Unidad de Medicina Reproductiva de la Clínica Monteblanco
}

\author{
Daniela Solari B. ${ }^{1}$, Sonia Villa V. ${ }^{1}$, Juan Enrique Schwarze M. ${ }^{1,2}$, José Balmaceda . $^{1}$, \\ Claudia Albornoz G.3, Ricardo Pommer T. 1,4 \\ 1 Unidad de Medicina Reproductiva de Clínica Monteblanco, ${ }^{2}$ Departamento Clínico de Obstetricia y Ginecología, Uni- \\ versidad de Santiago. ${ }^{3}$ Plastic Surgery Department, Memorial Sloan Kettering Cancer Center, New York. ${ }^{4}$ Instituto de \\ Investigación Materno Infantil, Universidad de Chile. Santiago, Chile.
}

\section{RESUMEN}

Objetivos: Determinar las motivaciones de mujeres para donar óvulos, y las significaciones que le otorgan a la misma. Método: Entrevista semiestructurada, cualitativa, a 18 mujeres candidatas a donantes de óvulos. Los tópicos analizados incluyeron: experiencias pasadas, fantasías y expectativas en relación a la ovodonación. Resultados: Las edades de las entrevistadas fluctuaron entre 19 y 29 años; 13 eran solteras y 6 tenían al menos un hijo. Más de la mitad estaban estudiando y trabajando al momento de la entrevista. Ninguna tenía antecedente de patología psiquiátrica personal ni familiar. La compensación económica fue la principal motivación para donar, aunque la empatía con las parejas infértiles fue otro importante motivador. Las potenciales donantes refirieron que el anonimato como protector para la donante y la pareja receptora. Las donantes percibieron la maternidad más relacionada con la crianza que con el vínculo genético. Conclusiones: Encontramos que las candidatas para ovodonar perciben la maternidad en función de la crianza y no del vínculo genético. Por lo tanto, asimilaron la donación de ovocitos con la donación de cualquier órgano. Sin embargo, manifiestan su rechazo a tener contacto con la familia receptora. Como era de esperar, la motivación económica fue la principal razón para donar, sin embargo, sentían fuerte empatía con las parejas infértiles.

PALABRAS CLAVE: Altruismo, actitudes, infertilidad, entrevista como tópico, donación de ovocitos

\section{SUMMARY}

Aims: To identify the motivating factors of candidates to donate oocytes, and the significance they assign to oocyte donation. Methods: We evaluated 18 volunteers trough a semi-structured interview. Topics discussed included: past experiences, fantasies and expectations towards the process of oocyte donation. Results: The ages fluctuated between 19 and 29 years; 13 were single and 6 had one or more children. More than a half were studying and working at the moment of the interview. None had familiar or personal psychiatric disorders. The economical compensation was the most important reason for the intention to donate, though the empathy with infertile couples was a significant motivator too. Potential donors perceived anonymity as protective for both donor and receptor. They perceived motherhood as rising the child rather than having a genetic link. Conclusions: We found that potential donors perceive motherhood as linked to rising a child, 
rather than having a genetic link. Therefore, they assimilate oocyte donation to the donation of any other organ. However, they prefer to have no contact with the oocyte receptor. As expected, the main motivator is the economical compensation, however they have strong feelings of empathy towards infertile couples.

\section{KEY WORDS: Altruism, attitudes, infertility, interviews as topic, oocyte donation}

\section{INTRODUCCIÓN}

Por más de veinte años la donación de óvulos ha permitido a miles de mujeres superar la infertilidad debido a la ausencia o mala calidad de los ovocitos remanentes. La ausencia de ovocitos se puede deber a enfermedades que dañen la reserva ovárica, exposición a agentes tóxicos como tabaco y drogas antineoplásicas, o ser el resultado natural del envejecimiento $(1,2)$. En otros casos, algunas parejas podrían recurrir a la utilización de ovocitos donados como una forma de evitar la transmisión materna de enfermedades genéticas.

En 1983, se reportaron los primeros casos de embarazo tras la transferencia de embriones producto de la fecundación de ovocitos donados $(3,4)$. La vitrificación de ovocitos permitió un nuevo avance en esta técnica, al conseguir disociar el ciclo ovárico de la donante del endometrial de la receptora, facilitando así la coordinación del procedimiento $(5,6)$.

Rápidamente esta técnica se expandió por el mundo, aumentando su demanda por pacientes que hasta entonces estaban condenadas a la esterilidad. Por ejemplo, en Latino América, el número de transferencias de embriones producto de ovodonación experimentó un aumento de más de $400 \%$ en los últimos diez años, de 1.471 en el año 2000 a 5.908 en el 2009 (7). Desde un comienzo ha existido un importante debate en torno a las distintas aristas asociadas a esta técnica. Dentro de las aristas que se discuten están quienes deben/pueden donar ovocitos, el anonimato, la motivación, y la retribución económica.

Está claro que muchos de las creencias y decisiones de las posibles donantes de óvulos están influenciadas por la sociedad en que viven. Así es como en Alemania, más de la mitad de los encuestados estaba a favor de la legalización de la donación de óvulos (8). Sin embargo, dentro de un país puede haber diferencias entre distintos grupos étnicos. Es así como en mujeres británicas de origen sudasiático eran menos favorables a donar ovocitos que sus pares caucásicas (9). Por otra parte, en Turquía solamente el $23 \%$ de los encuestados aprobaba la legalización de la donación de óvulos (10). En países musulmanes, tanto hombres como mujeres ven más favorablemente la donación de ovocitos que de espermios, a pesar que ambos son prohibidos por la ley islámica (11). Esto se traduce que en un determinado país la disponibilidad de donantes de gametos refleja el clima cultural más que la capacidad médica del país (12). Los aspectos culturales y sociológicos son determinantes importantes en las motivaciones y expectativas de las personas. Por esto no es fácil extrapolar los resultados de investigaciones realizadas en otros países, con otros valores culturales.

El objetivo del presente estudio es describir los significados que otorgan a la donación de óvulos las mujeres que desean participar en un programa de ovodonación. Para eso buscamos describir la motivación de las candidatas para donar; describir la significación que otorgan las candidatas a la ovodonación; describir los temores que sienten las candidatas a la ovodonación; describir la percepción de las candidatas a la eventual relación genética con un hijo/a que no conocerá; describir el concepto de maternidad de las candidatas; y describir la percepción frente al anonimato.

\section{MATERIAL Y MÉTODO}

Se realizó un estudio cualitativo, analizando las vivencias, motivaciones y expectativas de mujeres candidatas para donar ovocitos. Todas las donantes son entrevistadas por una psicóloga como rutina en nuestra unidad, de modo que todas las donantes fueron incorporadas en el estudio.

Dada la metodología cualitativa no se requirió muestreo probabilístico, sino que se realizó muestreo propositivo según las necesidades del estudio. El tamaño muestral fue determinado por saturación, es decir cuando dejaron de aparecer temas emergentes. Inicialmente estimamos que realizaríamos 20 entrevistas.

Se realizaron entrevistas semi-estructuradas por un miembro del equipo de investigación entrenado en metodología cualitativa. Los temas más relevantes analizados fueron: motivación para donar, significación de la donación, temores frente a la donación, importancia del lazo genético en la maternidad, relevancia del anonimato y concepción 
de la maternidad. Se diseñó una guía para la entrevista basada en revisión de la literatura, opinión de pacientes y discusión con expertos. La entrevista no excedió una hora de duración.

La recolección de los datos y el análisis se realizaron en forma conjunta, de manera de poder realizar cambios en el formato de las entrevistas de acuerdo a los temas emergentes, con el objetivo de capturar la máxima información. Las transcripciones fueron examinadas en detalle por al menos 2 miembros del equipo, con el objetivo de obtener una visión de los temas importantes emergentes para las parejas infértiles. Se realizó codificación línea a línea para examinar, comparar y desarrollar dominios y categorías conceptuales.

\section{RESULTADOS}

Se entrevistaron 18 candidatas para donación de óvulos. Las edades de las participantes fluctuaron entre 19-29 años, con una moda de 26 años. Trece pacientes estaban solteras al momento de la entrevista, 4 convivían y 1 casada. De ellas, 12 no tenían hijos al momento de la entrevista, y 6 tenían un hijo. Una de ellas había tenido un aborto espontáneo.

En cuanto a su nivel educacional, 4 de las participantes tenían educación superior completa, 8 superior incompleta, 3 técnica completa y 2 técnica incompleta. Una voluntaria no registró su nivel educacional. Al momento de la entrevista, 7 de las participantes estaban estudiando, y 2 de ellas además trabajaban al mismo tiempo. Ocho participantes estaban trabajando al momento de la entrevista, 2 se encontraban cesantes y 1 no registró la información.

Ninguna de las participantes tenía antecedentes familiares de patología psiquiátrica. La mayoría no manifestó problemática sicológica personal, una participante manifestó haber presentado poco tiempo antes de la entrevista una depresión reactiva frente a un duelo familiar, fenómeno que al momento de la entrevista se encontraba en regresión.

Motivaciones para donar. Las motivaciones para donar más frecuentemente comentadas fueron: económica (18 participantes) y ayudar a una pareja con problemas de fertilidad (12 participantes). Las participantes refirieron: "Es ayuda mutua, yo los ayudo y ellos me ayudan a mí"; "La compensación económica es importante, pero lo que más me motiva es el poder ayudar a alguien"; "Me importa mucho la ayuda que se le puede dar a parejas que no pueden tener"; "Por la felicidad de tener un bebé porque yo soy madre, además he visto lo que ha sufrido una amiga, además está la motivación económica"; "Necesito la plata, pero encuentro un acto muy bonito el darle la posibilidad a otra mujer de ser mamá".

Significación atribuida a la donación. La mayoría de las participantes consideraron la donación de óvulos similar a la donación de un órgano (17 participantes), atribuyéndole características altruistas por la posibilidad de ayudar a parejas infértiles. Las ideas fuerzas más repetidas eran: "Si puedo donar algo en vida es gratificante"; "Es como donar sangre, no creo que sea mi hijo porque no lo criaré"; "Yo soy donante de sangre y de órganos, donar óvulos es donar una célula que potencialmente puede ser un hijo, pero faltan cosas para que lo sea"; "El óvulo ni siquiera está fecundado, es hijo en proyecto, es la misma sensación que donar órganos, además se desperdician"; "No estoy dando un hijo, sino un óvulo, que es como donar un órgano"; "No están fecundados, los boto todos los meses, no los he gestado, así que no los considero como hijos sino como un órgano cualquiera que se puede donar". Una participante la refiere como un regalo de vida, "para mi donar óvulos es regalar vida".

Temores frente a la donación. De las 10 participantes que manifestaron temores frente a la donación de óvulos, la mayoría eran temores en relación a los procedimientos quirúrgicos y médicos relacionados con la estimulación y obtención de los óvulos. Al recibir mayor información sobre estos aspectos, las participantes manifestaron haber aclarado estos temores. Las frases que refieren a este respecto fueron: "Al principio me daba susto lo que me iban a hacer en el pabellón, con la anestesia y eso, pero al hablar con mi prima que ya donó y con el doctor, se me pasó"; "Me daba susto que esto tuviera consecuencias en mi salud a futuro, pero consulté a dos ginecólogos además del doctor del programa y ellos aclararon mis dudas y temores"; "Me daba susto quedar infértil después, pero aunque eso pasara, la adopción es una opción para mí"; "Me da susto cómo sacan los óvulos y lo de la anestesia"; "Los pinchazos es lo que más susto me da".

Opinión sobre el anonimato. Quince de las participantes consideró el anonimato como un factor positivo en el mecanismo de donación, algunas agregan que es un factor protector tanto para ellas como para la familia que recibe la donación, ya que los desvincula. Las opiniones más comúnmente mencionadas fueron: "El anonimato es ideal para mí, porque quiero donar y cerrar el proceso"; "Por respeto a la otra familia no me quiero incluir en su proceso, además quedan todos los parámetros bien claros"; "Me acomoda que sea anónimo, me siento protegida"; "Es bueno que sea anónimo, porque los protege a ellos y 
me protege a mi"; "Yo me pongo en el lugar de ellos y creo que me sentiría incómoda al conocerme"; "Es súper bueno para mi y para ellos, no voy a tener relación con ellos ni con la guagua que pudieran tener por la donación"; "Creo que es más importante para los receptores ya que así no se sentirán amenazados en el vínculo que desarrollarán".

Opinión sobre el vínculo genético con un posible hijo/a. Diecisiete de las participantes refiere no otorgarle ningún significado especial a la relación genética con el niño/a resultante de la donación. Consideraban que era un desconocido, esto tendría que ver con el concepto de maternidad que tiene la mayoría de las donantes. Las opiniones fuerza más repetidas fueron: "No me resulta complicado porque la guagua va a ser de la pareja, no mía"; "No me complica porque es sólo una célula"; "No creo que está donando un hijo, si así lo creyera no donaría, el óvulo es sólo una posibilidad de vida"; "No me resulta complicado porque aunque tenga una parte mía, no voy a tener un vínculo de gestación ni crianza con ese ser"; "No me complica, la guagua no va a ser mía, espero que los receptores lo cuiden"; "Desde el momento en que doné el óvulo, deja de ser mío y lo de la genética para mi no es tan importante"; "Mucho más importante que lo genético, son los lazos que uno crea con sus hijos".

Concepto de maternidad. Todas las participantes consideran como parte fundamental de la maternidad la crianza, 9 consideran además como parte muy importante de la maternidad la gestación. Una de las participantes agrega como tercer elemento de importancia en la maternidad, la genética. En este sentido, muchas de las pacientes con hijos la refieren que más importante que la genética es la crianza y el amor que se les da a los hijos. Los comentarios más repetidos fueron: "La maternidad está relacionada con la crianza y la formación de vínculos, estar en la trayectoria del bebé"; "La maternidad tiene que ver con la crianza, la formación de vínculos y estar en la trayectoria del bebé"; "Es la responsabilidad de salir adelante por alguien, ganas de protegerlo, enseñarle"; "Parte desde el primer vínculo que uno puede generar, la relación que se genera"; "Para mí la maternidad es tenerlo en la guatita, darle amor"; "Lo típico, la gestación, la crianza y lo genético".

Postura frente a parejas infértiles. Todas las participantes sentían empatía y se manifiestan muy sensibles con el tema de la infertilidad. Sentían que la donación era un acto filantrópico, la mayoría considera la ayuda a parejas infértiles como un motivo primordial para realizar la donación. Las ideas fuerza que encontramos fueron: "Siento empatía por las parejas infértiles porque he tenido casos cercanos";
"Yo creo que el hecho de ser mamá me influye para ayudar a otra mujer a vivir esa experiencia y hacerla feliz"; "Tengo una amiga que ha sufrido mucho con la infertilidad"; "Soy súper sensible con el tema porque tengo una amiga que tuvo una hija gracias a la donación"; "Lo siento como un problema cercano, porque tengo una tía que no pudo tener hijos y sufrió mucho".

\section{DISCUSIÓN}

Este el primer reporte nacional que describe las motivaciones y significaciones de mujeres que desean donar ovocitos. Además de los aspectos técnicos (hiperestimulación ovárica controlada y prevención del síndrome de hiperestimulación), la donación de ovocitos presenta varios desafíos a los centros de medicina reproductiva y al equipo multidisciplinario. Entre otras la adecuada selección de las mujeres que participan como donante, quienes deben donar, cuales son las características que deberían presentar, anonimato, compensación económica, etc.

Encontramos que al igual que en otros países, la motivación económica y el deseo de ayudar a parejas infértiles fueron los principales motivadores para donar óvulos. En Estados Unidos, donde la gran mayoría de las donaciones son voluntarias y compensadas económicamente, la mayoría de las donantes expresan satisfacción posdonación, y están dispuestas a repetir la donación (13). Las donantes consideran que la compensación económica es una justa retribución por los malos ratos pasados, y muchas no donarían sino hubiese una compensación económica (9). Algo similar ocurre en Argentina; en un estudio que incluyó 23 donantes voluntarias que contestaron un cuestionario estructurado, dentro de los primeros doce meses de realizada la donación, el $65 \%$ contestó que lo hizo por la motivación económica y $52 \%$ por razones altruistas (14). De hecho, en el Reino Unido donde está prohibido la compensación económica por donar ovocitos, existe una importante falta de donantes, por lo que muchos centros han recurrido a una solución intermedia. En esta solución intermedia, la donante debe ser una paciente con diagnóstico de infertilidad y requerimiento de fecundación in vitro, la que es subsidiada en parte o totalmente en los procedimientos que necesita como parte del tratamiento de su infertilidad conyugal, a cambio de donar una parte de los ovocitos recuperados $(9,15)$.

La donación de ovocitos es realizada principalmente por mujeres que sienten que no tienen un lazo de maternidad con el óvulo donado. Para las entrevistadas en este estudio, la maternidad se 
relacionaba más con la crianza de los niños que con la correspondencia genética, es decir, con el vinculo o relación que se genere y desarrolle con ese niño/a. Dentro de este paradigma de la maternidad, la donación de óvulos equivale a la donación de tan solo una célula. Sin embargo, a pesar de esto, las entrevistadas manifestaron que el anonimato era fundamental. Esto concuerda con la experiencia de Estados Unidos, donde muchas de las donantes no lo habrían hecho si se hubiera entregando información acerca de su identidad (15-17).

\section{CONCLUSIÓN}

El percibir la maternidad en función de la crianza y no de la correspondencia genética, las posibles donantes no sienten conflictos con entregar óvulos a desconocidos. Si bien la posibilidad de una compensación económica, por el tiempo perdido y las molestias propias del tratamiento, es el principal motivador para donar ovocitos, la empatía que sienten por las parejas infértiles es también un fuerte motor para la donación. Finalmente, el anonimato es percibido como una forma de protección para la donante y para la pareja receptora.

\section{REFERENCIAS}

1. Gracia CR, Sammel MD, Freeman E, Prewitt M, et al. Impact of cancer therapies on ovarian reserve. Fertil Steril 2012;97:134-40.e1.

2. Somigliana E, Benaglia L, Vercellini $P$, Paffoni $A$, et al. Recurrent endometrioma and ovarian reserve: biological connection or surgical paradox? Am J Obstet Gynecol 2011;204:529.e1-529.e5.

3. Buster JE, Bustillo M, Thorneycroft I, Simon JA, et al. Non-surgical transfer of an in-vivo fertilised donated ovum to an infertility patient. Lancet 1983;1:816-7.

4. Buster JE, Bustillo M, Thorneycroft IH, Simon JA, et al. Non-surgical transfer of in vivo fertilised donated ova to five infertile women: report of two pregnancies. Lancet 1983;2:223-4.

5. Cai LB, Qian XQ, Wang W, Mao YD, et al. Oocyte vitrification technology has made egg-sharing donation easier in China. Reprod Biomed Online 2012;24:18690.

6. Trokoudes KM, Pavlides C, Zhang X. Comparison outcome of fresh and vitrified donor oocytes in an eggsharing donation program. Fertil Steril 2011;95:19962000.

7. Zegers-Hochschild F, Schwarze JE, Crosby J, do Carmo M. Twenty years of assisted reproductive technology (ART) in Latin America. J Bra Assist Reprod 2011;15:15-30.

8. Stöbel-Richter Y, Goldschmidt S, Brähler E, Weidner K, Beutel M. Egg donation, surrogate mothering, and cloning: attitudes of men and women in Germany based on a representative survey. Fertil Steril 2009;92:124-30.

9. Purewal S, van den Akker OB. British women's attitudes towards oocyte donation: ethnic differences and altruism. Patient Educ Couns 2006;64:43-9.

10. Baykal B, Korkmaz C, Ceyhan ST, Goktolga U, Baser I. Opinions of infertile Turkish women on gamete donation and gestational surrogacy. Fertil Steril 2008;89:817-22.

11. Isikoglu $\mathrm{M}$, Senol $\mathrm{Y}$, Berkkanoglu $\mathrm{M}$, Ozgur $\mathrm{K}$, et al. Public opinion regarding oocyte donation in Turkey: first data from a secular population among the Islamic world. Hum Reprod 2006;21:318-23.

12. Braverman AM, Corson SL. Factors related to preferences in gamete donor sources. Fertil Steril 1995;63:543-9.

13. Kenney NJ, McGowan ML. Looking back: egg donors' retrospective evaluations of their motivations, expectations, and experiences during their first donation cycle. Fertil Steril 2010;93:455-66.

14. Glujovsky D, Fernandez L, Urdapilleta L, Sueldos S, et al. Assessment of motivations, attitudes and psychosocial features among voluntary oocyte donors in Argentina. Fertil Steril 2006;86:s56.

15. Purewal S, Vanden Akker O. 'I feel like they were mine and I should be looking after them': an exploration of non-patient women's attitudes towards oocyte donation. J Psychosom Obstet Gynaecol 2009;30:215-22.

16. Craft I, Thornhill A. Would 'all-inclusive' compensation attract more gamete donors to balance their loss of anonymity? Reprod Biomed Online 2005;10:301-6.

17. Craft I, Flyckt S, Heeley G, Layland S, et al. Will removal of anonymity influence the recruitment of egg donors? A survey of past donors and recipients. Reprod Biomed Online 2005;10:325-9. 